\title{
The prevalence and correlates of hallucinations in a general population sample: findings from the South African Stress and Health Study
}

\author{
H Temmingh', DJ Stein'1, S Seedat², DR Williams ${ }^{3}$ \\ ${ }^{1}$ Department of Psychiatry and Mental Health, University of Cape Town, South Africa \\ 2MRC Unit on Stress and Anxiety Disorders, Department of Psychiatry, University of Stellenbosch, Cape Town, South Africa \\ Harvard University School of Public Health, Boston MA, USA
}

\begin{abstract}
Objective: Large epidemiological surveys conducted in the developed world have found rates of psychotic symptoms in the general population to be as high as 10-28\%. However, there are few data available from developing countries, including African countries, on the prevalence and correlates of psychotic symptoms. This study investigates the prevalence and correlates of psychotic symptoms (ie hallucinations) in a general population sample of South African adults. Method: As part of the South African Stress and Health Study the prevalence of auditory and visual hallucinations was determined in a large community based sample of 4250 participants utilizing the Composite International Diagnostic Interview (CIDI). In addition, socio-demographic and clinical correlates as well as indicators of service utilization and functional impairment were determined. Results: The prevalence of any reported hallucination was $12.7 \%$, a rate comparable to that found in studies from the developed world. Multivariate analyses revealed a significant association between role impairment, service utilisation, suicidality and reported auditory or visual hallucinations. No significant association was found between urbanicity and reported psychotic symptoms. Conclusion: Our finding that psychotic symptoms (ie hallucinations) are significantly associated with functional impairment and service utilization supports the potential clinical significance of such symptoms, even in the African context.
\end{abstract}

Key Words: Prevalence; Psychosis; Population groups; Epidemiology; South Africa

Received: 23-12-2009

Accepted: 27-07-2010

doi: http://dx.doi.org/10.4314/ajpsy.vl4i3.4

\section{Introduction}

Psychotic experiences in non-patient populations have received increasing attention in recent years. ${ }^{1,2}$ Findings from large community-based epidemiological surveys from the United States of America (NCS-R, and ECA), Australia, the Netherlands (NEMESIS study) and Britain, have found rates of broadly defined psychosis phenotypes to range from 5\% to $28 \%{ }^{3-8}$ Other studies with samples of college students, primary care patients and the

\section{Correspondence}

Dr. H Temmingh

Department of Psychiatry and Mental Health, University of Cape Town,

Valkenburg Hospital, Private Bag X1, Observatory Cape Town, 7935

email: Henk.Temmingh@uct.ac.za general population have demonstrated that psychotic experiences are surprisingly common and occur in up to 50\% of participants. ${ }^{1,9}$ Some have argued in favour of a continuum or dimensional model for psychotic symptoms. ${ }^{10}$ Evidence exists of similarities in psychotic symptoms between clinical and non clinical samples, lending support to the notion that these symptoms represent the same underlying pathological process. These similarities include correspondence in demographic distribution, associated risk factors, homologous symptom structures as well as familial and longitudinal associations between schizotypal traits and the development of clinically defined psychosis phenotypes. ${ }^{1,10}$

In contrast to the developed world little is known regarding the prevalence of psychotic-like experiences and symptoms 
within the general population in developing countries including African countries such as South Africa. In a small study conducted in Uganda, delusion-like experiences were found to be more common than in samples from developed countries and were associated with urban residence even after controlling for confounders such as age, gender and social class. ${ }^{11}$ In the recent Nigerian Survey of Mental Health and Wellbeing (NSMHW), comprising of a sample representing $22 \%$ of the total Nigerian population, the CIDI 3.0 psychotic screen was administered to a smaller subsample of 1419 participants. The lifetime prevalence of non-affective psychotic symptoms was found to be $2.1 \%$ with visual hallucinations being the most frequent psychotic symptom, occurring in $1.2 \%$ of participants. ${ }^{12}$ The Nigerian study also found a significant association between reported psychotic symptoms and urbanicity as well as impaired role functioning. Findings from these two developing countries of an association between urbanicity and psychotic symptoms correspond to findings from developed countries such as the Netherlands and the United States of America. ${ }^{3,13}$ Studies from the developed world have also indicated that the presence of psychotic symptoms in community samples is associated with significantly more role impairment, higher rates of service use and hospitalization as well as increased rates of suicidal ideation. ${ }^{4,9}$ These findings need further replication in large representative community based samples within the African context.

The South African Stress and Health Study (SASH) is a large, nationally representative, community-based epidemiological survey that investigates mental disorder prevalence, indicators of disability, perceptions of stigma and exposure to trauma and stress. ${ }^{14}$ The lifetime prevalence of non-psychotic psychiatric disorders was found to be $30.3 \%$, with substance use disorders reaching a lifetime prevalence of $13.4 \% .^{15}$ These rates fall between the higher prevalence rates typically found in developed Western countries and lower rates from other African countries such as Nigeria. ${ }^{16-18}$ This study represents the first nationally representative survey utilizing a structured lay administered clinical interview to determine the prevalence of psychotic-like symptoms in the general population within Africa. We determined the presence of psychotic symptoms in the form of auditory or visual hallucinations and examined the associations and correlates of these symptoms with demographic variables like urban residence, ethnicity, level of education as well as indicators of impairment such as role interference and perceived health. Furthermore we examined associations between hallucinations and suicidal ideation as well as patterns of service utilization and hospitalization.

\section{Methods}

\section{Sample}

SASH methods are described elsewhere in detail. ${ }^{14}$ Adult participants (18 years or older) were selected according to a 3 stage, stratified, national probability sample. In the first stage census enumeration areas (EA's) from the 2001 South African Census of Enumeration Areas Survey were stratified according province, locality (rural vs. urban) and population group (African, Coloured, White, Indian). Designation of these groups is not intended to reify them, but rather is an attempt to approach the effects of historical classifications on current health disparities. A total of 960 EA's were selected randomly from these strata with the number of EA's selected per stratum proportional to the estimated stratum population. In order to maximize the inclusion of young working-age male participants, single-sex hostels where migrant labourers reside were also included. The second stage involved the selection of households within these geographic areas. In the third stage inhabitants were selected from each household and approached for an interview. The overall response rate was high and $85.5 \%$ agreed to participate. The psychotic screen was administered to a total sample 4250 participants. All racial and ethnic groups were included.

\section{Data collection}

Participants were administered the WHO Composite International Diagnostic Interview version 3.0 (CIDI 3.0). ${ }^{19}$ Since the aim of the study was to determine the prevalence of common mental disorders, complete diagnostic assessments for psychotic disorders were not included. The CIDI contains a series of screening questions for psychotic symptoms. In the SASH study the psychotic screen was modified to include only questions on screening for auditory and visual hallucinations. Each screening question was followed up by 4 questions relating to exclusion of symptoms in the context of altered levels of consciousness, substance related symptoms, and recording of the frequency and content of symptoms. Each reported symptom was followed up by 8 questions enquiring about various symptom characteristics. These included questions about age at onset, frequency, persistence, course, treatments from a medical or mental health practitioner and whether these symptoms lead to hospitalization. This report is limited to participants who endorsed any item examining the occurrence of lifetime auditory or visual hallucinations judged as not attributable to substances or altered levels of consciousness. No follow up diagnostic re-appraisal interviews were conducted.

Suicidal ideation was determined by the CIDI section for suicidality. This section assesses the presence of serious suicidal ideation in the participants' lifetime.

Role interference was determined by a series of questions from the screening module determining overall health and functioning and the section of the CIDI interview that assesses past 30 day functioning and disability. This section determines whether participants were unable to fulfil their normal role obligations in a social as well as occupational context for any amount of time.

Service use and overnight hospitalization was determined by the CIDI section for the assessment of service use and relevant sub-items from the psychotic screen.

Overall health status as perceived by participants is assessed within the screening section of the CIDI and consists of a 5 point self rating scale that determines participant's perception of general physical and mental health. For the purpose of this study overall self rated health was dichotomized into poor and fair categories.

Data collection was conducted by lay interviewers trained in centralized group sessions over a l-week period. Interviewers received specialized training in psychiatric interview techniques and two formal pre-tests of all the interview questions were completed. Only interviewers who mastered the administration of the interview were allowed to continue in the project. Interviews were conducted face to face at the participant's home in several different languages: (English, Afrikaans, Zulu, Xhosa, Sotho, and Tswana). The CIDI was translated and back translated into these languages by multilingual language experts. Following an iterative translation 
and back translation process, discrepancies in the backtranslation were discussed by an expert panel and the final format of the interview agreed upon. On average interviews were 3-4 hours in duration and often required more than one visit to complete. The protocol was approved by the ethics and human subject committees of the University of Michigan and Harvard Medical School and the Medical University of South Africa (MEDUNSA). All participants gave informed consent.

\section{Data analysis}

SASH person level data was weighted in order to account for differential probability of selection within the multistage stratified design and to account for non-response. Poststratification weighting was used to render the sample distribution comparable to the population distribution in the 2001 South African Census for age, sex and province. Psychotic symptoms were dichotomized into a group with one or more reported auditory or visual hallucinations and group with no reported auditory or visual hallucinations. Various measures of health outcome relating to service use, overnight

hospitalization, suicidal ideation, role interference and self rated health were dichotomized as dependent variables and entered into five different logistic regression models with various sociodemographic covariates and the presence of hallucinations as predictor variables. Socio-demographic predictor variables were: gender, age, race, marital status, years of education, employment status, urban/rural, and income.

Two socioeconomic status (SES) measures were included assets and wealth/debt status. Assets were calculated as a count of the total number of seven household appliances (refrigerator, vacuum cleaner, television, hi-fi or music centre, microwave oven, washing machine and video-cassette recorder) and seven household resources (running water, domestic servant, automobile, flush toilet, built-in kitchen sink, electric stove or hotplate, and working telephone) that respondents owned/ employed; as well as three financial activities that they engaged in (shopping at supermarkets, using financial services such as a bank account, automatic teller machine card or credit card, and having an account or credit card at a store). The alpha for this scale was 0.92 overall ( 0.89 for Blacks, 0.89 for Coloureds, 0.74 for Indians and 0.70 for Whites). Wealth was assessed by respondents reporting whether there would be any money left over if all their assets were sold and all their debts paid. Respondents reporting some wealth were contrasted with those reporting no or negative wealth and those who refused to provide an answer or indicated that they did not know the answer.

Categorical nominal variables for demographic groupings and educational level were utilized in bivariate analyses whereas educational level was entered as a continuous variable within the logistic regression models. Chi- square tests were used to analyse sociodemographic correlates of psychosis, with significance tests within multivariate logistic regression models calculated by Wald chi- square tests. Logistic regression coefficients and their design corrected standard errors are reported as odds ratios and 95\% confidence intervals (CIs). Significance levels of 0.05 were used throughout with all tests two sided and adjusted for the weighting and clustering of the data using the Taylor series linearization method. Data analysis was carried out on SAS and SAS-callable SUDAAN version 8.2 software. ${ }^{20}$

\section{Results}

\section{Sample characteristics}

Of the total sample of 4250 participants, 60.1\% ( $\mathrm{n}=2555)$ were female (Table I). Half of the participants $(50.2 \%, n=2315)$ were below 35 years of age. The majority of participants were black African (76\%, n=3232). Half of the participants (50.6\%, $\mathrm{n}=2154$ ) were not married, 69.7\% were unemployed, and 36.9\% were earning below 1500 ZAR per month. Most participants resided in urban areas $(57.9 \%, \mathrm{n}=2463)$ and $63.8 \%(\mathrm{n}=2712)$ had less than 12 years of education.

\begin{tabular}{|c|c|c|c|}
\hline & & \multicolumn{2}{|c|}{ Any auditory or visual hallucinations } \\
\hline & & No & Yes \\
\hline $\begin{array}{l}\text { Total } \\
\text { N } \\
\text { Percent }\end{array}$ & $\begin{array}{l}N \\
4250 \\
87.3\end{array}$ & $\begin{array}{l}3714 \\
12.7\end{array}$ & 536 \\
\hline $\begin{array}{l}\text { Gender } \\
\text { Male } \\
\text { Female } \\
\text { Chisq(p) }\end{array}$ & $\begin{array}{l}1695 \\
2555\end{array}$ & $\begin{array}{l}88.1(1.0) \\
86.7(0.8)\end{array}$ & $\begin{array}{l}11.9(1.0) \\
13.3(0.8) \\
192)\end{array}$ \\
\hline $\begin{array}{l}\text { Race } \\
\text { Black } \\
\text { Coloured } \\
\text { White } \\
\text { Indian } \\
\text { Chisq(p) }\end{array}$ & $\begin{array}{l}3232 \\
555 \\
305 \\
158\end{array}$ & $\begin{array}{l}86.2(0.9) \\
92.6(1.7) \\
89.5(3.4) \\
89.7(3.4)\end{array}$ & $\begin{array}{l}13.8(0.9) \\
7.4(1.7) \\
10.5(3.4) \\
10.3(3.4) \\
031)\end{array}$ \\
\hline $\begin{array}{l}\text { Age } \\
\begin{array}{l}18-34 \\
35-49 \\
50-64 \\
65+ \\
\text { Chisq(p) }\end{array}\end{array}$ & $\begin{array}{l}2135 \\
1248 \\
630 \\
237\end{array}$ & $\begin{array}{l}88.0(1.0) \\
86.5(1.4) \\
85.8(1.7) \\
89.9(2.3)\end{array}$ & $\begin{array}{l}12.0(1.0) \\
13.5(1.4) \\
14.2(1.7) \\
10.1(2.3) \\
524)\end{array}$ \\
\hline $\begin{array}{l}\text { Marital Status } \\
\text { Unmarried } \\
\text { Married } \\
\text { Chisq(p) }\end{array}$ & $\begin{array}{l}2154 \\
2096\end{array}$ & $\begin{array}{l}86.3(1.0) \\
88.3(1.0)\end{array}$ & $\begin{array}{l}13.7(1.0) \\
11.7(1.0) \\
118)\end{array}$ \\
\hline $\begin{array}{l}\text { Education } \\
\text { None } \\
\text { Grade 1-7 } \\
\text { Grade 8-11 } \\
\text { Grade 12 } \\
\text { Grade 13+ } \\
\text { Chisq(p) }\end{array}$ & $\begin{array}{l}323 \\
872 \\
1517 \\
913 \\
625\end{array}$ & $\begin{array}{l}87.9(2.3) \\
86.1(1.6) \\
85.6(1.2) \\
87.9(1.5) \\
91.8(1.2)\end{array}$ & $\begin{array}{l}12.1(2.3) \\
13.9(1.6) \\
14.4(1.2) \\
12.1(1.5) \\
8.2(1.2) \\
004)\end{array}$ \\
\hline $\begin{array}{l}\text { Income } \\
0 \\
1-1500 \\
1501-16500 \\
16501-97500 \\
97501+ \\
\text { Chisq(p) }\end{array}$ & $\begin{array}{l}607 \\
963 \\
895 \\
909 \\
876\end{array}$ & \begin{tabular}{|l|}
$85.6(2.2)$ \\
$86.5(1.3)$ \\
$89.6(1.2)$ \\
$86.2(1.3)$ \\
$88.2(1.3)$
\end{tabular} & $\begin{array}{l}14.4(2.2) \\
13.5(1.3) \\
10.4(1.2) \\
13.8(1.3) \\
11.8(1.3) \\
077)\end{array}$ \\
\hline $\begin{array}{l}\text { Employment } \\
\text { Unemployed } \\
\text { Employed } \\
\text { Chisq(p) }\end{array}$ & $\begin{array}{l}2964 \\
1286\end{array}$ & $\begin{array}{l}86.3(0.8) \\
89.6(1.1)\end{array}$ & $\begin{array}{l}13.7(0.8) \\
10.4(1.1) \\
006)\end{array}$ \\
\hline $\begin{array}{l}\text { Location } \\
\text { Rural } \\
\text { Urban } \\
\text { Chisq(p) }\end{array}$ & $\begin{array}{l}1787 \\
2463\end{array}$ & $\begin{array}{l}86.9(1.5) \\
87.6(0.9)\end{array}$ & $\begin{array}{l}13.1(1.5) \\
12.4(0.9) \\
697)\end{array}$ \\
\hline
\end{tabular}




\section{Relationship between psychotic symptoms and demographic characteristics}

In the total sample of 4250 participants, 12.7\% endorsed one or more items on the CIDI psychotic screen for hallucinations. A comparison of those with and without hallucinations (Table I) indicates that participants reporting any hallucinations on the CIDI interview were significantly more likely to unemployed and have lower levels of education and to be Black There was a trend towards significance for lower income groups to report more psychotic symptoms in the form of hallucinations. There was no association between urban residence, age, gender or marital status and reported hallucinations.

\section{Psychotic symptoms and health outcomes}

Bivariate analysis revealed a significant association between the presence of hallucinatory experiences and increased service use $\left(\mathrm{X}^{2}=12.2, \mathrm{df}=1, \mathrm{P}<0.001\right)$ as well as rates of overnight hospitalization ( $\mathrm{X}^{2}=10.3 \mathrm{df}=1, \mathrm{P}=0.002$ ). Significant associations were also found between the presence of hallucinations and reported suicidal ideation $\left(\mathrm{X}^{2}=16.6, \mathrm{df}=1, \mathrm{P}<0.001\right)$ and measures of impairment and disability such as role interference
$\left(\mathrm{X}^{2}=23.1, \mathrm{df}=1, \mathrm{P}<0.001\right)$ as well as perceived poor overall health status ( $\left.\mathrm{X}^{2}=52.9, \mathrm{df}=1, \mathrm{P}<0.001\right)$. Within all the multiple logistic regression models, with the exception for suicidal ideation, age was significantly associated with more service use, rates of hospitalization as well as greater role interference and poor health (Table II). Whereas Indian participants were less likely to report suicidal ideation, Black participants were more likely to report poor overall health. Employment and higher levels of education was associated with a significantly lower likelihood of role interference and perceived poor health, whereas and higher income was associated with lower rates reported of suicidal ideation. The presence of any reported hallucinations significantly predicted group membership for all the health outcomes in all the logistic regression models. In contrast to other variables, endorsement of hallucinatory experiences was consistently associated with significantly worse health outcomes across all the logistic regression models. As regards the magnitude of the association between hallucinations and the various health outcomes, odds ratios for all outcome categories were significant, with a particularly strong association with hallucinations and the likelihood of overnight hospitalisation (Table II).

\begin{tabular}{|c|c|c|c|c|c|}
\hline Variables & $\begin{array}{l}\text { Service Use } \\
N=4184\end{array}$ & $\begin{array}{l}\text { Overnight Hospital } \\
N=4184\end{array}$ & $\begin{array}{l}\text { Suicide Ideation } \\
N=3995\end{array}$ & $\begin{array}{l}\text { Role Interference } \\
N=3708\end{array}$ & $\begin{array}{l}\text { Health Fair/Poor } \\
N=4160\end{array}$ \\
\hline Female & $1.14(0.9-1.4)$ & $0.99(0.7-1.4)$ & $1.27(0.9-1.7)$ & $1.33(1.0-1.8)$ & $1.38(1.1-1.7)^{\star \star}$ \\
\hline Age & $1.02(1.0-1.0)^{\star \star \star}$ & $1.02(1.0-1.0)^{\star \star}$ & $0.99(1.0-1.0)$ & $1.02(1.0-1.0)^{\star \star \star}$ & $1.04(1.0-1.0)^{\star \star \star}$ \\
\hline \multicolumn{6}{|l|}{ White(reference) } \\
\hline Black & $1.29(0.7-2.5)$ & $1.21(0.4-3.3)$ & $0.68(0.4-1.2)$ & $1.01(0.4-2.7)$ & $1.90(1.0-3.6)^{\star}$ \\
\hline Coloured & $0.85(0.4-1.7)$ & $1.14(0.4-3.3)$ & $1.16(0.8-1.8)$ & $1.11(0.4-2.8)$ & $1.62(0.9-2.9)$ \\
\hline Indian & $1.51(0.7-3.3)$ & $0.58(0.2-2.2)$ & $0.46(0.3-0.8)^{\star \star}$ & $0.76(0.3-2.1)$ & $1.31(0.6-2.7)$ \\
\hline Log of Income & $1.00(0.9-1.1)$ & $1.01(0.9-1.2)$ & $0.90(0.8-1.0)^{\star \star}$ & $0.98(0.9-1.0)$ & $1.03(1.0-1.1)$ \\
\hline Married & $1.18(0.9-1.5)$ & $1.72(1.0-3.0)^{\star}$ & 1.03(0.8-1.3) & $0.93(0.7-1.2)$ & $1.00(0.8-1.2)$ \\
\hline Years of Education & $1.01(1.0-1.1)$ & $0.97(0.9-1.0)$ & $0.96(0.9-1.0)$ & $0.95(0.9-1.0)^{\star}$ & $0.93(0.9-1.0)^{\star \star \star}$ \\
\hline Employed & $0.98(0.8-1.2)$ & $0.38(0.2-0.7)^{\star \star}$ & $0.89(0.6-1.3)$ & $0.80(0.6-1.1)$ & $0.69(0.6-0.8)^{\star \star \star}$ \\
\hline Urban & $0.93(0.7-1.2)$ & $0.97(0.6-1.6)$ & $1.33(1.0-1.8)$ & $1.17(0.9-1.5)$ & $1.16(0.9-1.5)$ \\
\hline Other Assets & $1.06(1.0-1.1)^{\star *}$ & $1.10(1.0-1.2)^{\star \star}$ & 1.02(1.0-1.1) & $0.99(1.0-1.0)$ & $0.96(0.9-1.0)^{*}$ \\
\hline \multicolumn{6}{|l|}{ Wealth(reference) } \\
\hline Debt & $1.11(0.8-1.6)$ & $0.79(0.4-1.7)$ & $1.20(0.8-1.9)$ & $1.02(0.7-1.4)$ & $1.45(1.0-2.0)^{\star}$ \\
\hline Wealth UK/Ref/miss & $1.01(0.8-1.3)$ & $1.05(0.6-2.0)$ & $0.96(0.7-1.4)$ & $0.79(0.6-1.1)$ & $1.36(1.0-1.8)^{*}$ \\
\hline Psychosis Symptoms & $1.84(1.4-2.5)^{\star \star \star}$ & $3.28(1.9-5.6)^{\star \star \star}$ & $2.44(1.7-3.5)^{\star \star \star}$ & $2.41(1.8-3.2)^{\star \star \star}$ & $1.98(1.6-2.4)^{\star \star \star}$ \\
\hline \multicolumn{6}{|c|}{$\begin{array}{l}{ }^{*}=(\mathrm{p}-\text { value }<=.05) \quad \quad{ }^{* *}=(\mathrm{p}-\mathrm{value}<=.01) \quad \quad{ }^{* *}=(\mathrm{p}-\mathrm{value}<=.001) \\
\text { a. Auditory or visual hallucinations }\end{array}$} \\
\hline
\end{tabular}




\section{Discussion}

The results from this large community-based survey on the prevalence of psychotic symptoms in the form of visual or auditory hallucinations revealed that $12.7 \%$ of participants reported experiencing one or more hallucinations in their lifetime as assessed by the psychotic screen module of the CIDI. A number of studies have examined psychotic-like experiences within the general population in the developed world. Studies from the United States of America (NCS and NCS-R), Australia and the Netherlands (NEMESIS) investigating the broader psychosis phenotype utilising lay interviewers and the CIDI have found rates of psychotic symptoms in the general population ranging from 9-28\%.3-6 Studies that have focused on the presence of hallucinations in particular include the USA Epidemiologic Catchment Area (ECA) study, which reported a 13\% lifetime prevalence of hallucinations in a general population sample, as assessed by the Diagnostic Interview Schedule (DIS). ${ }^{8}$ Other studies that have used a broader definition of psychotic symptoms have reported different rates of hallucinations. In the British National Survey of psychiatric morbidity, hallucinations were reported in $4.2 \%$ of participants as screened by the psychosis screening questionnaire. ${ }^{7}$ The USA NCS-R study found a 9\% prevalence of broadly defined psychotic symptoms with the most frequently endorsed items on the CIDI psychotic screen being auditory (6.4\%) and visual hallucinations (4\%). ${ }^{4}$ The prevalence of reported hallucinations was several fold higher than reported delusions and delusion-like ideas in the NCS-R sample. Thus, despite the use of somewhat different methodologies, data from the SASH study are remarkably consistent with the ECA data, and correspond to other studies in that prevalence of reported hallucinations is far higher than prevalence of psychotic disorders such as schizophrenia. We report a much higher rate of hallucinations than found in the Nigerian survey (NSMHW). ${ }^{12}$ This may be due to methodological differences such as sampling differences and the use of an expert preliminary review in the Nigerian survey that may have lead to more conservative rates of nonaffective psychotic symptoms. Our sample also did not exclude affective psychosis. Our finding is consistent with the overall higher prevalence rates for other psychiatric disorders in the SASH study in comparison to the Nigerian survey results.

An interesting finding in our study is the significantly higher prevalence of hallucinations in Black participants. One possibility is that this is artefactual, reflecting the limitations of the survey methodology. Thus, the high rate of reported hallucinations in Black participants may simply reflect cultural idioms of distress or normative experiences, which are falsely endorsed as psychotic symptoms on the CIDI. Indeed, clinical re-appraisal in the NCS-R study found that up to $34.7 \%$ of visual and $29.5 \%$ of auditory hallucinations were reclassified as culturally appropriate and not psychotic. ${ }^{4}$ The fact that interviews were conducted in the participants' native language is likely to have reduced the impact of misinterpretation stemming from language related cultural effects. On the other hand the prevalence rate for hallucinations of $12.7 \%$ could represent an underestimation of the true rate of psychotic-like symptoms as we did not assess the presence of delusional ideation in our study.

Although we cannot rule out the possibility the findings here are artefactual, increased psychotic symptoms in Blacks possibly resonates with research from developed countries such as the Netherlands and the United Kingdom where social adversity associated with ethnic minority status and racial discrimination have been implicated as potential contributors to elevated rates of psychosis in non-western immigrant group. ${ }^{10,21}$ Hallucinatory experiences in particular have been shown to occur more frequently in Black than White ethnic groups in the UK and have been shown to be significantly associated with experiences of victimization. ${ }^{7,22}$ We have previously reported significantly higher rates of perceived racial discrimination among Black and mixed race participants in comparison to white participants within the SASH study population. ${ }^{23}$ As European immigrant populations are however likely to differ significantly from the South African population, further research on the relationship between psychotic phenomena and racial discrimination, victimization and social adversity within the South African context is needed.

Despite a comparatively similar prevalence rate of hallucinations in this population, several important differences exist between findings reported here and other populations. We could not demonstrate a significant relationship between younger age and higher rates of psychotic symptoms, a consistent finding in other studies investigating psychotic symptoms in the general population. A recent meta-analysis found a weak relationship between age and psychotic symptoms in the general population that only became apparent after taking into account smaller less methodologically robust studies. ${ }^{10}$ The fact that only hallucinatory experiences were assessed in our study could also explain the lack of association between age and psychotic experiences. Indeed, data from the ECA study revealed an increase of visual and auditory hallucinations with increased age. ${ }^{8}$ Possible confounding influences associated with age in the psychotic symptom group could include the presence of psychotic symptoms related to mood disorders, HIV or organic syndromes such as early dementia; as such conditions were not differentiated in the present analyses. As these conditions can all be associated with hallucinations and because they typically have later ages of onset in the case of dementia and psychotic mood disorders in females, or take years to develop, in case of advanced HIV illness with neurological sequelae, they could have influenced the association between age and psychotic symptoms.

In our sample, urban residential status was not associated with a higher rate of psychotic symptoms. This is in contrast to findings from other studies, including a small study from Uganda investigating delusion-like experiences as well as the Nigerian survey (NSMHW), 3,11,13 Reasons for this are unclear. Circular migration at a relatively advanced age and across the life cycle (past the high risk period for the development of psychosis) is common in the South African context. ${ }^{24}$ This could account for the lack of the association between urbanicity and psychosis prevalence. The fact that delusions were not assessed could have also led to the lack of relationship with urbanicity in our sample.

We could not demonstrate a significant relationship between male gender or single marital status and reported psychotic symptoms, a finding apparent across various studies as indicated by a recent meta-analysis. ${ }^{10}$ However 
studies investigating hallucinations in particular such as the ECA study reported the prevalence of hallucinations to be up to $50 \%$ higher in females. ${ }^{7,8}$ As we only assessed the presence of hallucinations in our study this could have accounted for the lack of male preponderance in our sample.

Important limitations of this study are the lack of (i) discrete assessment of schizophrenia and other psychotic disorders and (ii) clinical re-appraisal interviews in order to validate the presence and severity of psychotic symptoms. Endorsement of psychotic symptoms may therefore represent false positives. Clinical re-appraisal interviews conducted as part of similar studies from the developed world have demonstrated that hallucinations in isolation as assessed by lay interviewers have a relatively low correlation with clinician generated psychotic disorder diagnosis, whereas the presence of hallucinations in combination with other psychotic symptoms such as delusions are more likely to be associated with clinically confirmed diagnoses of psychosis. ${ }^{4}$ In contrast to these findings other studies have shown a strong correlation in non-clinical samples between the occurrence of hallucinations and delusions ${ }^{25,26}$, and it is therefore possible to speculate that this would also be the case in our sample.

Participants in our sample who endorsed items on the psychotic screen for hallucinations were significantly more likely to experience impairments as manifested by role interference and lower levels of perceived health. Overnight hospital stay and service utilization were also more frequent in participants who reported psychotic symptoms, indicating that these symptoms were unlikely to be arbitrary, or normative but were accompanied by distress and consequent help seeking. An association with indicators of role impairment and hospitalisation also predicted clinically relevant psychosis in a number of studies that used reappraisal methods (NCS and NCS-R), 3,4,12 Thus, while the current study is unable to address the multiple factors that might be causally related to such impairment, it is not unlikely that clinically relevant psychosis plays a role.

Although screening of the general population for psychotic symptoms is considered premature in light of unacceptably high false positive rates, screening of persons with mental symptoms in crisis presenting to primary care and those who access mental health services have been suggested to be of value in identifying persons who may be experiencing prodromal and at- risk mental states for the development of psychotic disorders such a schizophrenia. ${ }^{27,28}$ In addition some studies of prospective cohorts with subclinical psychotic-like experiences have reported significantly raised conversion rates to clinically diagnosable psychosis. ${ }^{29,30}$ In the SASH sample, in addition to high rates of service use, overnight hospitalization, role impairment and lower self reported health, patients with reported hallucinations were at significantly higher odds of experiencing suicidal symptoms in comparison to those who did not. These findings could have implications for primary care practitioners as it suggests that these patients may come in contact with medical services. Within the South African context treatment for psychiatric disturbances are mostly provided in the general medical sector, with few patients passing the filter to secondary and tertiary level mental health services. ${ }^{31,32}$ This would suggest the value of careful screening at primary health care level. However, given the low correlation of isolated hallucinations with clinical diagnoses of psychosis as reported in some studies, this would suggest that the finding of hallucinations on screening may not necessarily represent an underlying psychosis phenotype but could potentially also include a wide array of psychiatric syndromes including cultural-bound expressions of psychological distress.

\section{Conclusion}

Despite the limitations of this study, reported symptoms of psychosis within this general population sample were significantly associated with functional impairments and service utilization. This supports the notion that these symptoms could potentially represent a less severe variant of clinically manifest psychosis, and lends some support to the notion of the continuum concept of psychosis. Furthermore this study forms the groundwork for future research investigating relationships between variables such as ethnicity, discrimination and psychosis. In addition, it paves the way for further research into the pathways and presentation of at- risk mental states key to understanding and aiding in the planning of early intervention in psychosis initiatives.

\section{Acknowledgments}

The South African Stress and Health study was carried out in conjunction with the World Health Organization World Mental Health (WMH) Survey Initiative. We thank the WMH staff for assistance with instrumentation, fieldwork, and data analysis. These activities were supported by the United States National Institute of Mental Health (R01MH070884), the John D. and Catherine T. MacArthur Foundation, the Pfizer Foundation, the US Public Health Service (R13-MH066849, R01-MH069864, and R01 DA016558), the Fogarty International Center (FIRCA R01-TW006481), the Pan American Health Organization, Eli Lilly and Company, Ortho-McNeil Pharmaceutical, Inc., GlaxoSmithKline, and Bristol-Myers Squibb. The South African Stress and Health Study was funded by grant R0l-MH059575 from the National Institute of Mental Health and the National Institute of Drug Abuse with supplemental funding from the South African Department of Health and the University of Michigan. A complete list of WMH publications can be found at http://www.hcp.med.harvard.edu.wmh

\section{References}

1. Johns LC, van Os J. The continuity of psychotic experiences in the general population. Clin Psychol Rev 2001;21 (8):1125-41.

2. Stip E, Letourneau G. Psychotic symptoms as a continuum between normality and pathology. Can J Psychiatry $2009 ; 54(3): 140-51$.

3. Kendler KS, Gallagher TJ, Abelson JM, Kessler RC. Lifetime prevalence, demographic risk factors, and diagnostic validity of nonaffective psychosis as assessed in a US community sample. The National Comorbidity Survey. Arch Gen Psychiatry 1996;53(11):1022-31.

4. Kessler RC, Birnbaum H, Demler $O$ et al. The prevalence and correlates of nonaffective psychosis in the National Comorbidity Survey Replication (NCS-R). Biol PSychiatry 2005 ;58(8):668-76.

5. Scott J, Chant D, Andrews G, McGrath J. Psychotic-like experiences in the general community: the correlates of CIDI psychosis screen items in an Australian sample. Psychol Med 2006 ;36(2):231-8.

6. van Os J, Hanssen M, Bijl RV, Ravelli A. Strauss (1969) revisited: a 
psychosis continuum in the general population? Schizophr Res $2000 ; 45(1-2): 11-20$

7. Johns LC, Cannon $M$, Singleton $N$ et al. Prevalence and correlates of self-reported psychotic symptoms in the British population. Br J Psychiatry 2004 ; 185:298-305.

8. Tien AY. Distributions of hallucinations in the population. SoC Psychiatry Psychiatr Epidemiol $1991 ; 26(6): 287-92$.

9. Olfson M, Lewis-Fernandez R, Weissman MM et al. Psychotic symptoms in an urban general medicine practice. Am J Psychiatry $2002 ; 159(8): 1412-9$

10. van Os J, Linscott RJ, Myin-Germeys I, Delespaul P, Krabbendam L. A systematic review and meta-analysis of the psychosis continuum: evidence for a psychosis proneness-persistence-impairment model of psychotic disorder. Psychol Med 2009 ;39(2):179-95.

11. Lundberg P, Cantor-Graae E, Kabakyenga J, Rukundo G, Ostergren PO. Prevalence of delusional ideation in a district in southwestern Uganda. Schizophr Res 2004 1;71(1):27-34

12. Gureje O, Olowosegun O, Adebayo K, Stein DJ. The prevalence and profile of non-affective psychosis in the Nigerian Survey of Mental Health and Wellbeing. World Psychiatry $2010 ; 9(1): 50-5$

13. van Os J, Hanssen M, Bijl RV, Vollebergh W. Prevalence of psychotic disorder and community level of psychotic symptoms: an urbanrural comparison. Arch Gen Psychiatry 2001 ;58(7):663-8.

14. Williams DR, Herman A, Kessler RC et al. The South Africa Stress and Health Study: rationale and design. Metab Brain Dis $2004 ; 19(1-2): 135-47$.

15. Stein DJ, Seedat S, Herman A et al. Lifetime prevalence of psychiatric disorders in South Africa. Br J Psychiatry 2008 ;192(2):112-7.

16. Gureje O, Lasebikan VO, Kola L, Makanjuola VA. Lifetime and 12 month prevalence of mental disorders in the Nigerian Survey of Mental Health and Well-Being. Br J Psychiatry 2006 ; 188:465-71.

17. Kessler RC, Berglund P, Demler O, Jin R, Merikangas KR, Walters EE Lifetime prevalence and age-of-onset distributions of DSM-IV disorders in the National Comorbidity Survey Replication. Arch Gen Psychiatry $2005 ; 62(6): 593-602$

18. Demyttenaere K, Bruffaerts R, Posada-Villa J et al. Prevalence, severity, and unmet need for treatment of mental disorders in the World Health Organization World Mental Health Surveys. JAMA 2004 2;291 (21):2581-90

19. Kessler RC, Ustun TB. The World Mental Health (WMH) Survey Initiative Version of the World Health Organization (WHO)

Composite International Diagnostic Interview (CIDI). Int J Methods
Psychiatr Res 2004;13(2):93-121

20. Research Triangle Institute. SUDAAN: Professional Software for Survey Data Analysis (computer program), 8.01 ed. Research Triangle Park, N.C. : Research Triangle Institute. 2002

21. Veling W, Selten JP, Susser E, Laan W, Mackenbach JP, Hoek HW. Discrimination and the incidence of psychotic disorders among ethnic minorities in The Netherlands. Int J Epidemiol $2007 ; 36(4): 761-8$.

22. Johns LC, Nazroo JY, Bebbington P, Kuipers E. Occurrence of hallucinatory experiences in a community sample and ethnic variations. Br J Psychiatry 2002 ; 180:174-8

23. Williams DR, Gonzalez HM, Williams S, Mohammed SA, Moomal H, Stein DJ. Perceived discrimination, race and health in South Africa. Soc Sci Med $2008 ; 67(3): 441-52$.

24. Lurie M. Migration and AIDS in southern Africa: a review. South African Journal of Science 96, 343-347. 2000.

25. Laroi F, Van der LM. Metacognitions in proneness towards hallucinations and delusions. Behav Res Ther 2005 ;43(11):1425-41.

26. Verdoux $H$, van OJ. Psychotic symptoms in non-clinical populations and the continuum of psychosis. Schizophr Res 2002 ;54(1-2):59-65.

27. Hanssen MS, Bijl RV, Vollebergh W, van OJ. Self-reported psychotic experiences in the general population: a valid screening tool for DSM-III-R psychotic disorders? Acta Psychiatr Scand $2003 ; 107(5): 369-77$.

28. Yung AR, Buckby JA, Cotton SM et al. Psychotic-like experiences in nonpsychotic help-seekers: associations with distress, depression, and disability. Schizophr Bull 2006 ;32(2):352-9

29. Bak $M$, Delespaul $P$, Hanssen $M$, de GR, Vollebergh $W$, van OJ. How false are "false" positive psychotic symptoms? Schizophr Res $2003 ; 62(1-2): 187-9$.

30. Poulton R, Caspi A, Moffitt TE, Cannon M, Murray R, Harrington H. Children's self-reported psychotic symptoms and adult schizophreniform disorder: a 15-year longitudinal study. Arch Gen Psychiatry $2000 ; 57(11): 1053-8$.

31. Williams DR, Herman A, Stein DJ et al. Twelve-month mental disorders in South Africa: prevalence, service use and demographic correlates in the population-based South African Stress and Health Study. Psychol Med 2008 ;38(2):211-20.

32. Seedat S, Stein DJ, Herman A et al. Twelve-month treatment of psychiatric disorders in the South African Stress and Health Study (World Mental Health Survey Initiative). Soc Psychiatry Psychiatr Epidemiol 2008 ;43(11):889-97.

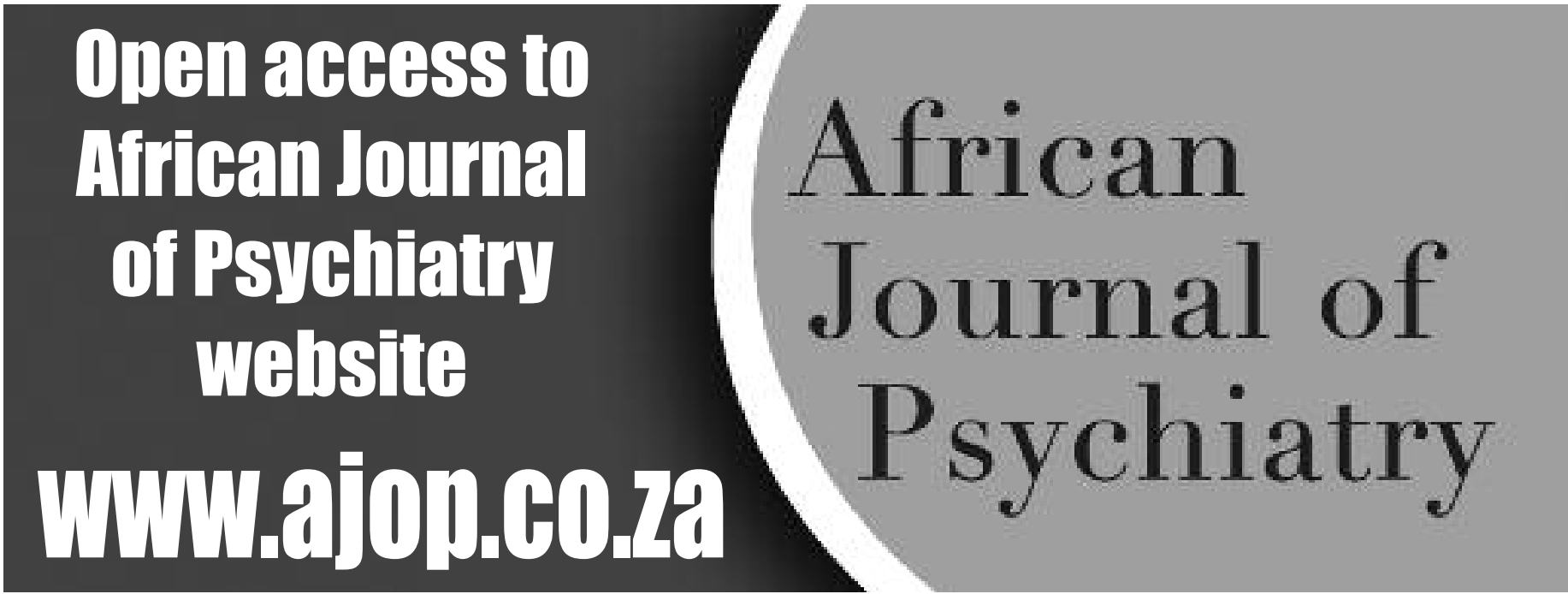

